\title{
A gas-jet apparatus for high-resolution laser spectroscopy on the heaviest elements at SHIP
}

\author{
S. Raeder ${ }^{\mathrm{a}, \mathrm{b}, *}$, M. Block ${ }^{\mathrm{a}, \mathrm{b}, \mathrm{c}}$, P. Chhetri ${ }^{\mathrm{d}, \mathrm{a}}$, R. Ferrer ${ }^{\mathrm{e}}$, S. Kraemer ${ }^{\mathrm{e}}$, T. Kron ${ }^{\mathrm{b}, \mathrm{a}}$, M. Laatiaoui ${ }^{\mathrm{b}, \mathrm{c}}$, S. Nothhelfer $^{\mathrm{c}}$, F. Schneider $^{\mathrm{b}, \mathrm{c}}$, \\ P. Van Duppen ${ }^{\mathrm{e}}$, M. Verlinde ${ }^{\mathrm{e}}$, E. Verstraelen ${ }^{\mathrm{e}}$, Th. Walther $^{\mathrm{d}}$, A. Zadvornaya ${ }^{\mathrm{e}}$ \\ ${ }^{a}$ GSI Helmholtzzentrum für Schwerionenforschung, Planckstraße 1, 64291 Darmstadt, Germany \\ ${ }^{b}$ Helmholtz Institut Mainz, Johann-Joachim-Becherweg 36, 55128 Mainz, Germany \\ ${ }^{c}$ Johannes Gutenberg Universität Mainz, Johann-Joachim-Becher-Weg 45, 55128 Mainz, Germany \\ ${ }^{d}$ Technische Universität Darmstadt, Schloßgartenstraße 7, 64289 Darmstadt, Germany \\ ${ }^{e}$ KU Leuven, Instituut voor Kern- en Stralingsfysica, Celestijnenlaan 200D, B-3001 Leuven, Belgium
}

\begin{abstract}
Laser spectroscopy enables the determination of fundamental atomic and nuclear properties with high precision. In view of the low production rates of the heaviest elements, a high total efficiency is a crucial requirement for any experimental setup to be used in on-line experiments. The setup requires the use of gas stopping techniques to slow down the radionuclides of interest. In previous studies laser spectroscopy was performed inside a gas-filled stopping cell with a limited spectral resolution of a few GHz. Collisional broadening inside stopping cells ultimately limits the precision of laser spectroscopic studies and hampers in particular hyperfine spectroscopy. The spectral linewidth is reduced by an order of magnitude when the laser spectroscopy is performed in a well-collimated gas jet formed by the exit nozzle of a gas stopping cell. In addition, the exposure of the jet to high-repetition rate laser light which saturates the optical transitions allows maintaining a high total efficiency. Here, we present a new setup dedicated to laser spectroscopy of the heaviest elements with an improved resolution, which is presently under construction. This setup combines the efficient filament neutralization demonstrated for nobelium with the improved resolution of in-gas-jet spectroscopy.
\end{abstract}

Keywords: Resonance ionization spectroscopy, gas stopping cell, gas jet, nobelium

\section{Introduction}

Studying optical transitions by laser spectroscopy gives access to basic atomic and nuclear properties. While precise atomic structure data has been thoroughly documented for most 5 of species, a scarcity of such information still prevails the region of the heaviest elements. Besides the atomic structure, ${ }_{30}$ information related to the size and shape of the atomic nucleus is imprinted in subtle changes of the electron binding energy [1]. Due to the low production rates of a few ions per second at 10 most and half-lives which are shorter then a few minutes for the heaviest elements, the experimental exploration of their atomic structure is extremely challenging. For more than ten years, fermium (Fm, $Z=100$ ) was the heaviest element for which atomic levels had been identified [2]. However, recently laser spec15 troscopy on nobelium (No, $Z=102)$ was successfully performed at the in-flight separator SHIP at GSI [3] using the RAdia- ${ }_{40}$ tion Detection Resonance Ionization Spectroscopy (RADRIS) technique inside a buffer-gas stopping cell filled with about 100 mbar of argon gas [4, 5]. Besides the identification of 20 several atomic levels in nobelium, its first ionization potential was determined by precise investigations of Rydberg series [6]. 45 Moreover, the isotope shifts for the isotopes ${ }^{252-254}$ No were measured and a hyperfine splitting in ${ }^{253}$ No was observed from

*Email corresponding author: s.raeder@gsi.de which nuclear ground-state properties were inferred [7]. Espe25 cially in the latter case, the precision was mainly limited by the achieved spectral line width of about $3 \mathrm{GHz}$, which was insufficient to resolve all individual hyperfine components in ${ }^{253} \mathrm{No}$. For laser spectroscopy inside the gas cell, which was applied in the case of nobelium and fermium, the resolution is dominated by the laser bandwidth and more fundamentally by the gas-pressure-induced collisional broadening. A higher spectral resolution in the actinide region was obtained only for elements as heavy as plutonium $(\mathrm{Pu}, Z=94)$ in an atomic beam effusing from a hot atomizer as well as in collinear laser spectroscopy on a fast ion beam using macroscopic, ng-size samples in both cases [8]. The combination of a narrow spectral profile and a high total efficiency was achieved only recently for short-lived actinium isotopes by performing laser spectroscopy in a supersonic gas jet [9-11]. Here, the homogeneous low-pressure and low-temperature environment in the jet effusing from a stopping cell through a de Laval nozzle enables high resolution spectroscopy while the well-formed, parallel gas jet in conjunction with a high-power, $10 \mathrm{kHz}$ repetition rate laser system ensures a high efficiency of up to $10 \%$ [9, 12]. For actinium [11] a resolution of $\leq 400 \mathrm{MHz}$ (FWHM) was obtained, which is an improvement by about one order of magnitude compared to the results obtained with the RADRIS technique [3, 7]. In addition, the continuous operation of the gas jet enables the study of shorter-lived isotopes in comparison to the RADRIS tech- 


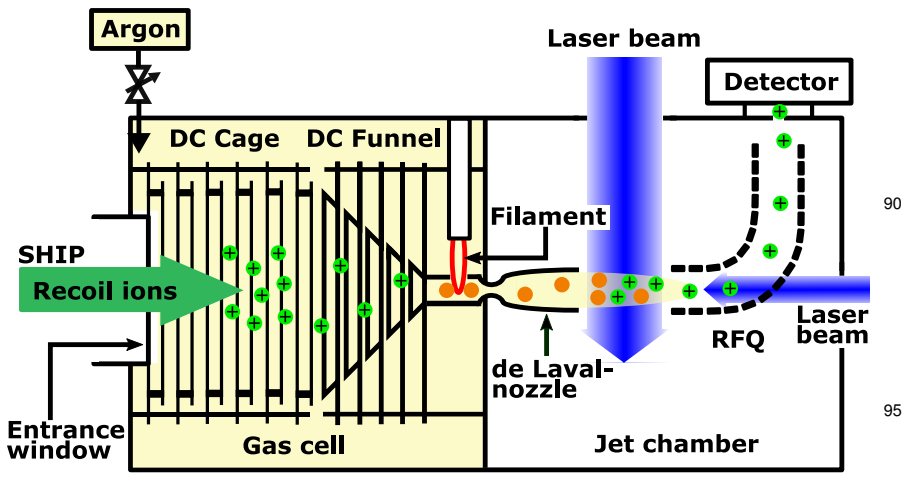

Figure 1: Concept of the gas cell for laser spectroscopy of heavy elements in a supersonic gas jet. The recoil ions (green) are thermalized in the gas cell and guided by electric fields to a filament for neutralization. The neutral atoms (orange) leave the cell through the nozzle and laser spectroscopy is performed by ${ }^{100}$ resonant ionization in the low-density and low-temperature gas jet. For details see text.

nique that features separated cycles of sample collection and spectroscopy.

In this article we present a concept of a setup dedicated to highresolution laser spectroscopy of the heaviest elements. The aim of this setup is to perform laser spectroscopy with a resolution of a few $100 \mathrm{MHz}$ on isotopes and isomers of heavy elements after suitable optical transitions have been identified with, e.g. the RADRIS technique. Besides an improved determination of nuclear moments and charge radii, this setup will allow for the provision of clean beams for, e.g. $\alpha$ - and $\gamma$-spectroscopic investigations. This is of particular interest as the $K$-isomers in this region of deformed nuclei are linked to the single particle configurations of even heavier systems. Starting from simulation studies to benchmark the performance of the system and to guide the technical construction, a gas-cell design is derived.

65 The final design of the gas cell and the current status is presented.

\section{Concept of the gas jet apparatus}

In the new gas cell setup, the RADRIS concept of ion $_{125}$ collection and neutral desorption from a heated filament is 70 combined with the gas-jet laser ionization. The conceptual design is shown in Fig. 1. We anticipate to use this setup at the Separator for Heavy Ion reaction Products (SHIP) [13] at GSI, Darmstadt. The setup consists of two main sections: the high- -130 pressure (30-100 mbar) gas cell for ion stopping and the jet chamber for laser spectroscopy. Initially, heavy and superheavy elements (SHE) are produced through fusion-evaporation reactions and are separated from the intense primary beam (up to $1 \mathrm{p} \mu \mathrm{A}$, corresponding to $6.25 \times 10^{12}$ particles/s) by SHIP. The ${ }_{135}$ high-energetic fusion-evaporation residues (recoils) enter the gas cell through a thin window that separates the argon-filled volume from the vacuum of the separator and, in addition, reduces the energy of the incoming recoils. Argon is used as buffer gas as it provides a larger stopping power compared to 140 helium, but also results in a suitable velocity of about $550 \mathrm{~m} / \mathrm{s}$ the argon gas and are guided towards a resistively heated tantalum filament by applying electrostatic voltages at the cage, comprising a set of cylindrical electrodes, at the funnel, which consists of electrodes with a decreasing diameter, and at the filament to better focus the ions. The large volume required for stopping the recoils results in a gas flow which is too slow to transport short-lived species without significant losses due to radioactive decay. Therefore, the electric field is used to enable a fast transport of the ions towards the filament from the large volume, which is required to stop the recoils. Details on the expected transport time will be discussed later in this article. A faster gas flow in this respect would require sophisticated and space-consuming pumping systems, which are not feasible at the experimental area, where several experimental setups, such as RADRIS, SHIPTRAP [14], and COMPASS [15] need to be installed at the focal plane of SHIP. Accordingly, the setup has to be compact to also enable a fast installation. The filament is located inside an exit channel in the vicinity of the extraction nozzle and heated to a temperature of about $1050^{\circ} \mathrm{C}$ at which nobelium desorbs as a neutral atom. This neutral desorption from tantalum was studied in detail for the RADRIS experiment [5, 16]. In the new setup, the evaporated neutral atoms are then transported by the gas flow towards a converging-diverging nozzle of de Laval type. The gas effusing from the nozzle will form a low-density and low-temperature gas-jet seeded with nobelium atoms, where the density is defined by the background pressure of $10^{-2}-10^{-1}$ mbar and the temperature depends on the machnumber and is about $50 \mathrm{~K}$ for a Mach 5 gas jet. By matching the pressure of the jet chamber with that of the jet at the nozzle exit a long collimated gas jet, suitable for high-resolution laser spectroscopy studies, will be formed [12]. The final mass flow in the setup is limited by the pumping capacity of the turbomolecular pump for the gas-jet chamber of about $1300 \mathrm{l} / \mathrm{s}$. From a preparatory test a maximum stagnation pressure of about $100 \mathrm{mbar}$ or $30 \mathrm{mbar}$ in the gas cell is expected for a nozzle throat diameter of $0.6 \mathrm{~mm}$ or $1 \mathrm{~mm}$, respectively. The laser beams are arranged in a cross beam geometry and interact with the atoms in the supersonic gas-jet performing two-step resonant laser ionization spectroscopy. For our studies on the heaviest elements we require the usage of a pulsed laser system with a repetition rate of at least $10 \mathrm{kHz}$ to cover all atoms in the emerging gas jet within the first $55 \mathrm{~mm}$ distance from the nozzle [17]. Such a laser system will not be discussed in this work and for simplicities sake, we refer to Ref. [17] in this respect, where a laser system similar to the one intended for usage at GSI is presented. Once the sample atoms are re-ionized by the laser beams, the photo-ions are captured and guided to a detector inside a detector chamber by a curved radio frequency quadrupole (RFQ). To achieve a high vacuum below $1 \times 10^{-4}$ mbar in the detector chamber, the latter is separated by a differential pumping stage from the jet chamber. A channel electron multiplier (CEM) can be used in off-line studies on long-lived isotopes. Alternatively, a silicon detector is anticipated for radiation detection while studying short-lived $\alpha$-decaying nuclides. 


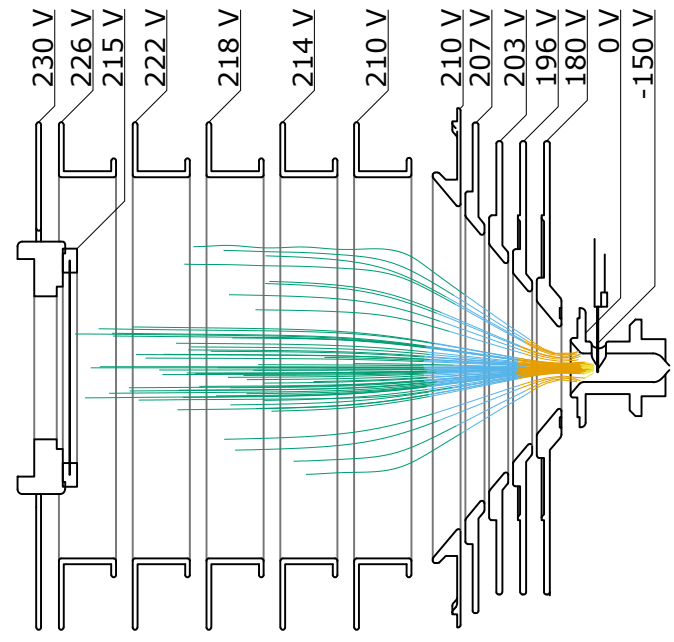

Figure 2: Simulated trajectories of 50 ions after thermalizing in the argon buffer gas. The voltages of the electrodes are given on the top, while the color of the trajectory represents the velocity of the ion (green $v_{\text {ion }} \leq 2 \mathrm{~m} / \mathrm{s}$, blue $v_{\text {ion }} \leq 15 \mathrm{~m} / \mathrm{s}$, orange $v_{\text {ion }} \leq 125 \mathrm{~m} / \mathrm{s}$, yellow $\left.v_{\text {ion }} \leq 1000 \mathrm{~m} / \mathrm{s}\right)$. Over $70 \%$ of the stopped ions were transported to the filament. For more details, see text.

\section{Simulation studies}

Starting from these general concepts and guided by simulations on the ion trajectories and on the gas flow dynamics inside the gas cell, a detailed layout of the setup was conceived. The ${ }_{175}$ main conclusions of these simulations will be briefly discussed.

\subsection{Ion trajectories}

After stopping and thermalizing the recoil ions in the buffer gas, electric fields will be used to guide the ions towards a fil- ${ }^{180}$ ant for neutralization. The main requirements are high accumulation efficiency and short transport time. Typical modern gas stopping cells use a combination of a DC gradient and a socalled RF funnel or carpet, a set of closely packed electrodes fed by an RF voltage to guide the ions into the extraction noz- ${ }^{18}$ zle throat as discussed, e.g., in Ref. [18]. In our case, the use of argon gas at a pressure of $\geq 30$ mbar prohibits the application of relatively large RF amplitudes of about $500 \mathrm{~V}$ needed for an efficient focusing of the low-mobility and heavy ions. Thus, solely DC gradients are used inside the gas cell. In the pres- ${ }^{190}$ ence of the argon buffer gas, the motion of the ions is damped such that their trajectories closely follow the electric field lines. The electrode design of the stopping cell was therefore chosen to contain a large diameter drift region, referred to as cage, in which the ions are fully stopped, and a converging region, re- ${ }^{195}$ ferred to as funnel, which provides the focusing field as well as a collimation of the gas flow.

The trajectories of the ions inside this cell were calculated using the SIMION software [19], including functions that add viscous damping based on ion mobilities in argon. The ions' initial start positions within the cell were obtained from SRIM 200 [20] simulations of the stopping positions of uranium ions with a kinetic energy of about $36 \mathrm{MeV}$ that were stopped in 30 mbar argon after traveling through a titanium foil with a thickness of $3.5 \mu \mathrm{m}$. Uranium is used because it is the heaviest ion for

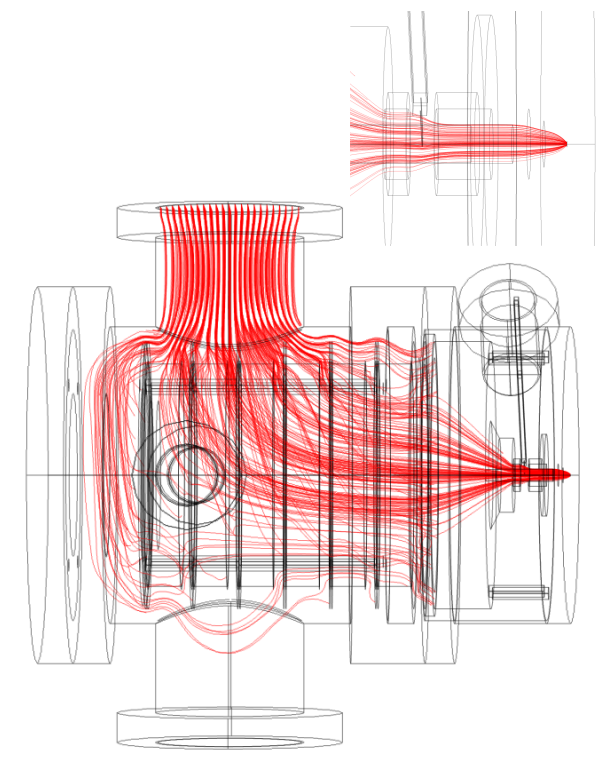

Figure 3: Streamlines of the argon flow in the gas cell. A homogeneous inflow from the top part is assumed. The flow is disturbed within the cage structure, while the ions are guided by electric fields. The exit channel features a cut-out to enable the placement of the filament. The inset shows the homogeneous gas flow in this exit channel towards the nozzle, where the neutralized evaporation residues are transported only by the gas flow.

which detailed stopping power data are available. The energy is matched to the recoil energy of nobelium produced in the fusion-evaporation reaction ${ }^{208} \mathrm{~Pb}\left({ }^{48} \mathrm{Ca}, 2 \mathrm{n}\right){ }^{254}$ No resulting from a $200 \mathrm{MeV}$ primary ${ }^{48} \mathrm{Ca}$ beam. Additional constrains for the ion trajectory simulations were given by the maximum voltages applicable to the individual electrodes before discharges may occur. These were approximated for a pressure of $30 \mathrm{mbar}$ from the Paschen law and the minimum distances between the electrodes and the cell wall.

Figure 2 shows the simulated trajectories for 50 ions projected on the symmetry plane. The voltages were refined to maximize the number of ions entering the flow channel surrounding the neutralization filament. A transport efficiency close to unity could not be achieved due to the large field gradient required close to the filament to keep up a point-charge like geometry. With the shown voltage set, $76 \%$ of the simulated ions were successfully transported to the filament, within a mean transport time of $95 \mathrm{~ms}$. To estimate diffusion-induced transport losses, the simulation code was extended to include hard-sphere collisions and diffusion models. The simulations indicated that even when accounting for diffusion, about $70 \%$ transport efficiency shall be achievable.

\subsection{Gas-flow simulations}

Numerical simulations of the argon gas flow inside the gas cell have been performed using the COMSOL Multiphysics ${ }^{\circledR}$ software [21] with the aim to optimize the gas-cell geometry for in-gas-jet laser spectroscopy experiments on nobelium isotopes. These experiments require a gas-cell geometry, which ensures a laminar flow through the small volume hosting the tantalum filament and the nozzle exit. This in turn will enable a 


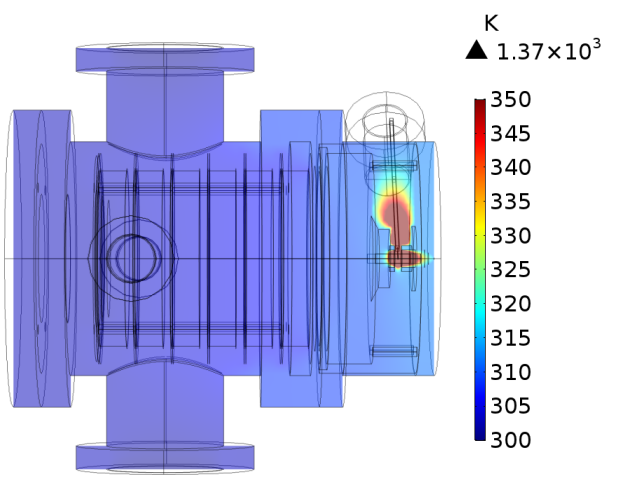

Figure 4: Equilibrium temperature profile in the central plane of the gas cell. mizing diffusion and decay losses and suppressing the influence of filament heating on the gas-jet temperature that impacts the spectral resolution.

The gas-cell design under consideration is shown in Fig. 3 The gas cell at a stagnation pressure of 30 mbar argon and a temperature of $300 \mathrm{~K}$ with an exit throat diameter of $0.6 \mathrm{~mm}$. The flow simulations used the compressible-flow physical model valid for Mach numbers $M<0.3$. Therefore, only the part with subsonic flow, e.g., until the converging part of the nozzle reaches270 an inner diameter of $1.2 \mathrm{~mm}$ was included (see Eq. 12 in Ref. [9] ). The inflow velocity was determined by conservation of the volume flow rate using Eq. 27 in Ref. [9]. In the simulations, a free tetrahedral mesh was implemented with a normal and an extremely fine element size for the body and exit region of the 275 gas cell, respectively.

The Laminar Flow package was used to study the influence of the large central-body volume and that of the gap for the filament holder on the flow conditions in the exit channel of the gas cell. The obtained streamlines, displayed in Fig. 3, show a 280 homogeneous laminar flow in the extraction region of the gas cell. The extraction time of the sample atoms from the filament to the nozzle exit was determined using the Particle Tracing for Fluid Flow package. One thousand nobelium atoms, simulated as spheres with an atomic radius of $200 \mathrm{pm}$, were randomly dis-285 tributed over the central part of the filament imposing the gasvelocity field as the initial velocity condition. These atoms undergo Brownian motion in time steps of $10^{-10}$ and $10^{-7} \mathrm{~s}$ and reach the nozzle exit in $56-67 \mathrm{~ms}$. air at atmospheric pressure except for the wall containing the exit hole which was set at 0.3 Torr. Heat transfer to the surroundings was also taken into account. The temperature of the $0.125 \mathrm{~mm}$ thick tantalum filament was fixed at $1373 \mathrm{~K}$ and that physics Nonisothermal Flow package was used to simulate the influence of the permanently heated filament on the stagnation temperature and the gas-jet temperature. This package couples the Laminar Flow and the Heat Transfer in Fluids packages equilibrium temperatures in the central plane of the gas cell are shown in Fig.4 At the nozzle exit the gas reaches $\sim 320 \mathrm{~K}$. For a Mach 8.5 nozzle, this increases the gas-jet temperature to $12.8 \mathrm{~K}$ instead of $12 \mathrm{~K}$ when not heating the filament (c.f. Eq. 6 in Ref. [9]). This corresponds to an increase in the expected spectral broadening from $138 \mathrm{MHz}$ to $144 \mathrm{MHz}$ (c.f. Eq. 15 in Ref. [9]) which means that the heating of the filament has only a negligible effect on the spectral resolution. From these simulations and in order to reduce the extraction time, it was decided to shorten the extraction region of the gas cell by $5 \mathrm{~mm}$ and to increase the gas velocity by reducing the diameter of the last cylindrical region of the exit channel. This adjustment increases the gas temperature to $325 \mathrm{~K}$ at the nozzle exit, resulting in an expected spectral resolution of $145 \mathrm{MHz}$, whereas the extraction time is reduced by a factor of two, i.e. down to 28-32 ms.

\section{Layout and construction}

The discussed gas-flow and ion-trajectory simulations guided the technical design of the gas cell and electrode structure in an iterative process, resulting in the final design shown in Fig.5 The dimensions of the stopping volume were inferred from SRIM simulations as discussed earlier, assuming a minimum gas pressure of $30 \mathrm{mbar}$ in the gas cell, which is the lower limit for operation in the case of a nozzle throat diameter of $1 \mathrm{~mm}$. With smaller nozzle throats higher pressures in the gas cell can be reached, which in turn will reduce the volume needed for stopping. The cage structure to which an electrostatic potential gradient is applied has a length of $164.5 \mathrm{~mm}$ and an inner diameter of $160 \mathrm{~mm}$ and is placed inside a CF250 vacuum chamber. The cage is followed by the funnel structure consisting of five electrodes of gradually decreasing diameters with a total length of $63.2 \mathrm{~mm}$. This structure guides the ions to the exit flow channel of $12 \mathrm{~mm}$ in diameter and a length of $34.6 \mathrm{~mm}$. The filament feedthrough is designed such that on final position the tip of the filament is on the central axis of the exit channel. The nozzle is designed in collaboration with KU Leuven where detailed studies on the nozzle shape and the characterization of the resulting gas jets are ongoing [12, 17]. The atoms in the gas jet are excited and ionized by laser radiation before they are captured and transported towards the detection system. To ease access to the gas jet for laser radiation and to improve differential pumping a $90^{\circ}$-bend RFQ structure is used for ion transport, similar to the structure used in Ref. [9].

To ensure low outgassing for reaching ultra-high vacuum conditions, the gas-cell chamber and all electrodes are made of 316L stainless steel. A 3001/s turbo molecular pump (Leybold, MAG W300) is used to evacuate the gas-cell volume. A valve with a metal sealing is used to separate the pump when the gas cell is operated with buffer gas. To ease handling, the gas-cell chamber is divided in two parts: one contains the entrance window and the cage and a second part contains the funnel. The second part also hosts the gas-jet chamber, separated by a wall welded inside the chamber. The gas-jet chamber has a length of $310 \mathrm{~mm}$. Here the curved RFQ structure is placed 50$68 \mathrm{~mm}$ behind the nozzle, depending on the nozzle in use. This 

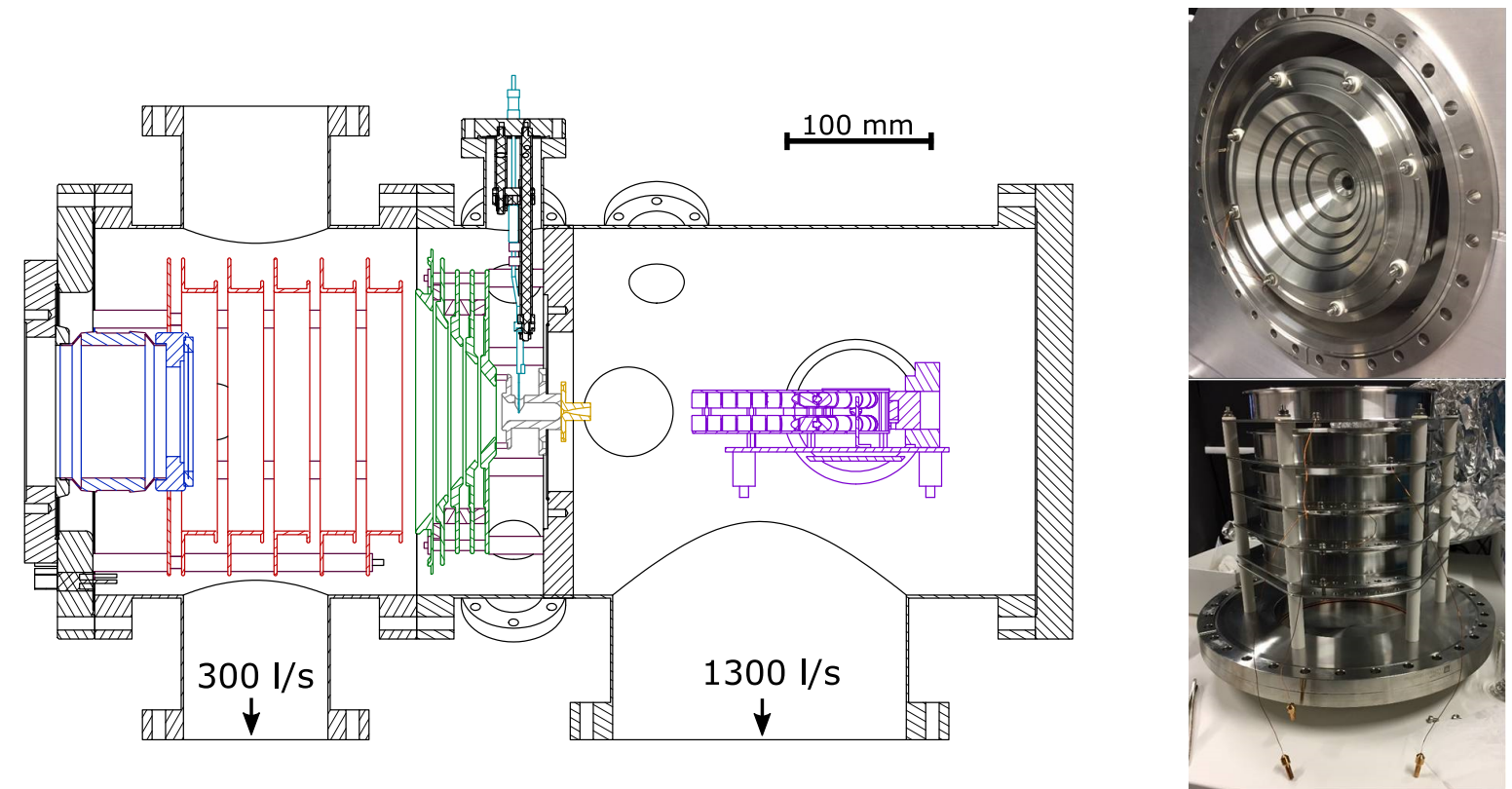

Figure 5: Left: technical drawing of the new setup. The gas cell (left volume) hosts from left to right the entrance window (blue), the cage (red), the funnel (green), the filament (cyan) and the nozzle (yellow). The gas jet chamber has a view port for the laser and a curved RFQ (purple) for ion extraction. For details see text. Right: Photographs of the funnel (top picture) and the cage structure (bottom picture) before installation.

chamber is equipped with a turbo molecular pump (Edwards STPH1303) for high gas throughput with a pumping capacity of $1300 \mathrm{l} / \mathrm{s}$. In front of the pump a butterfly valve is placed, which allows matching the background pressure for ideal jet formation [12]. For mounting of, e.g. the filament and the laser 335 entrance window as well as for electric connection and vacuum diagnostics, different CF100, CF63, and CF40 flanges are available in both chambers.

In first tests, pressures of $8.7 \times 10^{-8} \mathrm{mbar}$ and $1.8 \times 10^{-8} \mathrm{mbar}$ have been reached after 2 days of pumping in the gas-cell and the gas-jet chamber, respectively. To maintain a low level of impurities during gas operation it is intended to add a non evaporable getter (NEG) pump installed on one of the CF100 flanges of the gas cell. The buffer gas is argon of high purity (99.9999\% purity) which is additionally purified by a heated getter (SAES Pure Gases, Monotorr PS4-MT3) to the ppb level before it is injected into the gas cell. The ppb level of purity is required to avoid unwanted neutralization in the large stopping volume and any molecule formation from collisions with impurities during the extraction time. With this constellation we were able to operate the gas cell at 30 mbar as anticipated in on-line experiments for an extended period using a nozzle of $1 \mathrm{~mm}$ throat diameter.

\section{Summary and outlook}

A conceptual and technical design of a setup for laser spectroscopy of the heaviest elements in a gas jet emerging from $\mathrm{a}_{355}$ stopping cell is presented. This setup combines the neutralization of stopped ions from a heated filament with the gas flow extraction of the desorbed neutral atoms into the gas jet formed by a de Laval nozzle. The characterization and construction of the $e_{360}$ nozzles is performed at KU Leuven. First tests on the vacuum system and the electric components in the gas cell show that the vacuum level reached is sufficiently low and that the operation pressures of about 30 mbar in conjunction with a $1 \mathrm{~mm}$ nozzle matches the expected specification of the available pumping system. The next steps include the production and detection of laser ions as well as the characterization of the gas cell in terms of extraction efficiency and transport time.

\section{Acknowledgments}

This work was supported by the German Federal Ministry of Research under contracts 06MZ169I, 06LM236I, FAIR NuSTAR 05P09RDFN4, 05P12RDFN8, and 05P15RDFN1; and by the Helmholtz Association. This project has also received funding from the European Union Horizon 2020 research and innovation programme under the grant agreement no. 654002 (ENSAR2). This work has received funding from Research Foundation Flanders (FWO, Belgium), by GOA/2015/010 (BOF KU Leuven) and from the European Research Council (ERC-2011AdG-291561-HELIOS).

\section{References}

[1] P. Campbell, I. Moore, M. Pearson, Prog. Part. Nucl. Phys. 86 (2016) 127-180. doi 10.1016/j.ppnp.2015.09.003

[2] M. Sewtz, H. Backe, A. Dretzke, G. Kube, W. Lauth, P. Schwamb, K. Eberhardt, C. Grüning, P. Thörle, N. Trautmann, P. Kunz, J. Lassen, G. Passler, C. Z. Dong, S. Fritzsche, R. G. Haire, Phys. Rev. Lett. 90 (2003) 163002. doi 10.1103/PhysRevLett.90.163002

[3] M. Laatiaoui, W. Lauth, H. Backe, M. Block, D. Ackermann, B. Cheal, P. Chhetri, C. E. Düllmann, P. van Duppen, J. Even, R. Ferrer, F. Giacoppo, S. Götz, F. P. Heßberger, M. Huyse, O. Kaleja, J. Khuyagbaatar, P. Kunz, F. Lautenschläger, A. K. Mistry, S. Raeder, E. Mi- 
naya Ramirez, T. Walther, C. Wraith, A. Yakushev, Nature 538 (2016) 495-498. doi 10.1038/nature19345

[4] H. Backe, W. Lauth, M. Block, M. Laatiaoui, Nucl. Phys. A 944 (2015) 492 - 517. doi 10.1016/j.nuclphysa.2015.07.002

[5] F. Lautenschläger, P. Chhetri, D. Ackermann, H. Backe, M. Block, B. Cheal, A. Clark, C. Droese, R. Ferrer, F. Giacoppo, S. Götz, F. Heßberger, O. Kaleja, J. Khuyagbaatar, P. Kunz, A. Mistry, M. Laatiaoui, W. Lauth, S. Raeder, T. Walther, C. Wraith, Nucl. Instrum. Meth.

370 Phys. Res. B 383 (2016) 115-122. doi 10.1016/j.nimb.2016.06.

[6] P. Chhetri, D. Ackermann, H. Backe, M. Block, B. Cheal, C. Droese, C. E. Düllmann, J. Even, R. Ferrer, F. Giacoppo, S. Götz, F. P. Heßberger, M. Huyse, O. Kaleja, J. Khuyagbaatar, P. Kunz, M. Laatiaoui, F. Lautenschläger, W. Lauth, N. Lecesne, L. Lens, E. Minaya Ramirez, A. K. Mistry, S. Raeder, P. Van Duppen, T. Walther, A. Yakushev, Z. Zhang,

I. Phys. Rev. Lett. 120 (2018) 263003. doi 10.1103/PhysRevLett.120. 263003

[7] S. Raeder, D. Ackermann, H. Backe, R. Beerwerth, J. C. Berengut, M. Block, A. Borschevsky, B. Cheal, P. Chhetri, C. E. Düllmann, V. A. Dzuba, E. Eliav, J. Even, R. Ferrer, V. V. Flambaum, S. Fritzsche, F. Giacoppo, S. Götz, F. P. Heßberger, M. Huyse, U. Kaldor, O. Kaleja, J. Khuyagbaatar, P. Kunz, M. Laatiaoui, F. Lautenschläger, W. Lauth, A. K. Mistry, E. Minaya Ramirez, W. Nazarewicz, S. G. Porsev, M. S. Safronova, U. I. Safronova, B. Schuetrumpf, P. Van Duppen, T. Walther 385 C. Wraith, A. Yakushev, Phys. Rev. Lett. 120 (2018) 232503. doi 10. 1103/PhysRevLett.120.232503

[8] A. Voss, V. Sonnenschein, P. Campbell, B. Cheal, T. Kron, I. D. Moore, I. Pohjalainen, S. Raeder, N. Trautmann, K. Wendt, Phys. Rev. A 95 (2017) 032506. doi 10.1103/PhysRevA.95.032506

[9] Y. Kudryavtsev, R. Ferrer, M. Huyse, P. Van den Bergh, P. Van Duppen, Nucl. Instrum. Meth. B 297 (2013) 7-22. doi 10.1016/j .nimb. 2012. 12.008

[10] S. Raeder, B. Bastin, M. Block, P. Creemers, P. Delahaye, R. Ferrer, X. Fléchard, S. Franchoo, L. Ghys, L. Gaffney, C. Granados, R. Heinke, L. Hijazi, M. Huyse, T. Kron, Y. Kudryavtsev, M. Laatiaoui, N. Lecesne, F. Luton, I. Moore, Y. Martinez, E. Mogilevskiy, P. Naubereit, J. Piot, S. Rothe, H. Savajols, S. Sels, V. Sonnenschein, E. Traykov, C. V. Beveren, P. V. den Bergh, P. V. Duppen, K. Wendt, A. Zadvornaya, Nucl. Instrum. Meth. Phys. Res. B 376 (2016) 382-387. doi 10.1016/j.nimb. 2015.12.014 proceedings of the $\{$ XVIIth $\}$ International Conference on Electromagnetic Isotope Separators and Related Topics (EMIS2015), Grand Rapids, MI, U.S.A., 11-15 May 2015.

[11] R. Ferrer, A. Barzakh, B. Bastin, R. Beerwerth, M. Block, P. Creemers, H. Grawe, R. de Groote, P. Delahaye, X. Fléchard, S. Franchoo, S. Fritzsche, L. P. Gaffney, L. Ghys, W. Gins, C. Granados, R. Heinke, L. Hijazi, M. Huyse, T. Kron, Y. Kudryavtsev, M. Laatiaoui, N. Lecesne, M. Loiselet, F. Lutton, I. Moore, Y. Martnez, E. Mogilevskiy, P. Naubereit, J. Piot, S. Raeder, S. Rothe, H. Savajols, S. Sels, V. Sonnenschein, J.-C. Thomas, E. Traykov, C. Van Beveren, P. Van den Bergh, P. Van Duppen, W. K., A. Zadvornaya, Nature Commun. 8 (2017). doi $10.1038 /$ ncomms 14520

[12] A. Zadvornaya, P. Creemers, K. Dockx, R. Ferrer, L. Gaffney, W. Gins, C. Granados, M. Huyse, Y. Kudryavtsev, M. Laatiaoui, E. Mogilevskiy, S. Raeder, S. Sels, P. V. den Bergh, P. V. Duppen, M. Verlinde, E. Verstraelen, M. Nabuurs, D. Reynaerts, P. Papadakis, Phys. Rev. X 8 (2018). doi $10.1103 /$ physrevx.8.041008

[13] G. Münzenberg, W. Faust, S. Hofmann, P. Armbruster, K. Güttner, H. Ewald, Nucl. Instrum. Meth. 161 (1979) 65 - 82. doi 10.1016/ 0029-554X (79) 90362-8

420 [14] M. Block, D. Ackermann, K. Blaum, C. Droese, M. Dworschak, S. Eliseev, T. Fleckenstein, E. Haettner, F. Herfurth, F. Heßberger, et al., Nature 463 (2010) 785-788. doi 10.1038/nature08774

[15] D. Ackermann, A. Mistry, F. Heßberger, B. Andel, S. Antalic, M. Block, L. Caceres, P. Chhetri, F. Dechery, C. Droese, C. Düllmann, F. Giacoppo, J. Hoffmann, O. Kaleja, O. Kamalou, N. Kurz, J. Khuyagbaatar, R. Mändl, T. Murböck, M. Laatiaoui, J. Maurer, P. Mosat, J. Piot, S. Raeder, H. Savajols, O. Sorlin, C. Stodel, J. Thomas, M. Vostinar, P. Wieczorek, A. Yakushev, Z. Zhang, Nucl. Instrum. Methods Phys. Res., Sect. A 907 (2018) 81-89. doi 10.1016/j.nima.2018.01.096

16] M. Laatiaoui, H. Backe, M. Block, F.-P. Heßberger, P. Kunz, F. Lautenschläger, W. Lauth, M. Sewtz, T. Walther, Europ. Phys. J. D 68 (2014) 71 doi 10.1140/epjd/e2014-40617-6

[17] Y. Kudryavtsev, P. Creemers, R. Ferrer, C. Granados, L. Gaffney, M. Huyse, E. Mogilevskiy, S. Raeder, S. Sels, P. V. den Bergh, P. V. Duppen, A. Zadvornaya, Nucl. Instrum. Meth. Phys. Res. B 376 (2016) 345-352. doi 10.1016/j.nimb.2016.02.040

[18] C. Droese, S. Eliseev, K. Blaum, M. Block, F. Herfurth, M. Laatiaoui, F. Lautenschläger, E. M. Ramirez, L. Schweikhard, V. Simon, P. Thirolf, Nucl. Instrum. Methods Phys. Res., Sect. B 338 (2014) 126-138. doi 10. 1016/j.nimb.2014.08.004

[19] D. A. Dahl, International Journal of Mass Spectrometry 200 (2000) 3-25. doi $10.1016 / \mathrm{S} 1387-3806(00) 00305-5$

[20] J. F. Ziegler, Srim, Online, 2016. URL: www .srim.org

[21] Comsol multiphysics, 2018. URL:https://www.comsol.com/ 